\title{
As clear as mud: new evidence regarding transfusion triggers in cardiac surgery
}

\author{
Christopher Harle, MB, ChB (D) - Matthew A. Chong, MD
}

Received: 19 January 2020/ Accepted: 22 February 2020/Published online: 18 March 2020

(C) Canadian Anesthesiologists' Society 2020

In this edition of the Journal, Kashani et al. ${ }^{1}$ have contributed some additional insights into the already significant literature regarding restrictive $v s$ liberal blood transfusion in cardiac surgical patients. Why is this important, and why are we still asking the question?

In the current era of pervasive groupthink, echo chambers, and "fake news", it becomes increasingly important that we look to solid evidence emanating from high-quality science to guide our clinical practice. The many, however, rely on the few when it comes to understanding and employing statistical methods to interpret evidence, and, who knew-even statistics are themselves subject to statistics and interpretation. As such, tremendous responsibility lies with those entrusted with both discerning and disseminating facts within the constraints of reliable statistical methods.

The issue of transfusion triggers in cardiac surgery is a little more opaque than we might have thought after the Transfusion Requirements in Cardiac Surgery (TRICS) III ${ }^{2}$ and Transfusion Indication Threshold Reduction 2 (TITRe2) $^{3}$ trials-both of which recruited large numbers of patients. For instance, we suspect that most of us dismiss the statistically significant increase in all-cause mortality at

\footnotetext{
C. Harle, $\mathrm{MB}, \mathrm{ChB}(\bowtie)$

Department of Anesthesia and Perioperative Medicine, Western University, London, ON, Canada

e-mail: Christopher.harle@lhsc.on.ca
}

Department of Anesthesia \& Perioperative Medicine, University Hospital (London Health Sciences Centre), 339 Windermere Road, C3-108, London, ON N6A 5A5, Canada

\section{A. Chong, MD}

Department of Anesthesia and Perioperative Medicine, Western University, London, ON, Canada
90 days in the restrictive transfusion group of the TITRe2 trial. We do this on the basis that meta-analyses (the highest quality evidence) suggest that there is probably no difference between restrictive and liberal transfusion thresholds in 90-day mortality. We trust the metaanalyses, which incorporate data from all available trials, rather than simply "believing" the results of one individual trial. Similarly, we have probably accepted without much skepticism the consensus from the systematic reviews ${ }^{4-6}$ cited that unnecessary transfusion should be avoided because there is no strong evidence of harm in restrictive transfusion, without perhaps applying due diligence and asking the arguably more pertinent question: what is the evidence, if any, for improved outcomes with a restrictive transfusion strategy, and what is the risk? These questions are of course difficult to answer, not least of all because of the heterogeneity of the studies, patients, end points, and transfusion triggers used in the studies.

Although not the first meta-analysis on the topic of perioperative transfusion triggers in cardiac surgical patients, Kashani et al. ${ }^{1}$ are one of the first teams to utilize trial sequential analysis (TSA) as part of their metaanalysis. Although mathematically complex, the theory underlying TSA is simple: it is a cumulative meta-analysis that involves the creation of trial sequential boundaries for superiority, inferiority, or futility. If the meta-analytic result crosses the futility boundary, this suggests that further trials would not change the overall result of the meta-analysis (e.g., the research would be futile). Conversely, if the result crosses either of the superiority or inferiority boundaries, this suggests that sufficient information size (cumulative sample size) and statistical significance has been achieved for the results to be considered definitive. Finally, it may be the case that 
none of these thresholds are crossed and that further research has the potential to alter the meta-analytic result. ${ }^{7}$

Interestingly, despite the 9,101 patients included in the analysis done by Kashani et al., ${ }^{1}$ only $30 \%$ of the required information size was reached and none of the superiority, inferiority, or futility boundaries were crossed. From a clinician's perspective, this means that, on one hand, further trials could potentially show additional benefit insofar as mortality and the other secondary outcomes studied are concerned. On the other hand, any potential benefit (if any at all) is likely to be of small and perhaps of a clinically insignificant magnitude. Thus, the overall evidence-based take home message remains the same: perioperative patients receive fewer blood transfusions with a restrictive $v s$ liberal transfusion trigger-with no apparent detriment to clinical outcomes.

That unnecessary blood transfusion is risky is beyond question-we are all acutely aware of the potential negative clinical outcomes and the costs of blood products. Our understanding of this issue was shaped by the tainted blood scandals from the 1980's whereby thousands of patients were inadvertently infected with human immunodeficiency virus and/or hepatitis $\mathrm{C}$, which subsequently improved our understanding of the negative immunological outcomes associated with transfusion. So where does this leave us in seeking to practice evidencebased transfusion practice? The crucial question (and perhaps the Holy Grail) of this field of research would be to identify the actual trigger at which transfusion becomes beneficial to each individual patient. To have a personalized and contextual trigger where we know with certainty that there is a therapeutic advantage to transfuse is what we should strive for. Until then, we will probably all still unnecessarily transfuse some patients, and not transfuse some others who would benefit. Of course, a safe artificial hemoglobin substitute would nullify much of the angst associated with red cell transfusion, and perhaps that is the true Holy Grail.

This important study shows two things that are true to life and science in general. Sometimes we don't know what we don't know even when we think we know what we know. The standard conclusion to so much of our scientific literature that "more research is needed" is ubiquitous and frustrating to those who want the final answer. Perhaps this study takes this one step further and suggests that more research is unlikely to tell us the answer.

\section{Rien n'est moins clair: nouvelles données probantes à propos des seuils de transfusion en chirurgie cardiaque}

Dans ce numéro du Journal, Kashani et coll. ${ }^{1}$ ont apporté de nouveaux éléments à la littérature déjà abondante sur les seuils de transfusions sanguines restrictives $v s$ libérales en chirurgie cardiaque. Pourquoi est-ce important, et pourquoi nous posons-nous encore la question?

À l'ère actuelle de pensée unique, de matraquage médiatique et de fausses nouvelles, il est devenu essentiel de tenir compte de données probantes rigoureuses issues d'une science de la plus haute qualité qui soit afin de guider notre pratique clinique. La majorité, toutefois, s'appuie sur la minorité lorsqu'il s'agit de comprendre et d'employer des méthodes statistiques pour interpréter les données probantes et, oh surprise - les statistiques sont elles-mêmes soumises à des statistiques et de l'interprétation. Dès lors, une responsabilité phénoménale repose sur les quelques individus à qui l'on confie la tâche de distinguer et de disséminer les faits dans le cadre des contraintes imposées par les méthodes statistiques fiables.

La question des seuils de transfusion en chirurgie cardiaque est plus opaque qu'on aurait pu l'espérer après les études TRICS III $^{2}$ (Transfusion Requirements in Cardiac Surgery) et TITRe $2^{3}$ (Transfusion Indication Threshold Reduction 2), deux études ayant recruté un nombre important de patients. Par exemple, il est fort probable que la plupart d'entre nous ne tiennent pas compte de l'augmentation statistiquement significative de la mortalité toutes causes confondues à 90 jours dans le groupe de transfusion restrictive de l'étude TITRe2. Nous agissons ainsi en nous appuyant sur le principe que les méta-analyses (soit les données probantes de la plus haute qualité) suggèrent qu'il n'y a probablement pas de différence entre des seuils de transfusion libéraux ou restrictifs dans l'incidence de mortalité à 90 jours. Nous faisons confiance aux méta-analyses, qui intègrent les données de toutes les études disponibles, plutôt que de simplement «croire» les résultats d'une étude individuelle. De la même manière, nous avons probablement accepté sans grand scepticisme le 
consensus des revues méthodiques ${ }^{4-6}$ citées, selon lequel les transfusions non nécessaires devraient être évitées étant donné qu'il n'existe pas de données probantes convaincantes des conséquences négatives de seuil de transfusions restrictif et ce, sans peut-être avoir fait preuve de rigueur appropriée ni avoir demandé la question assurément plus pertinente: quelles sont les données probantes, si tant est qu'elles existent, de devenirs améliorés en cas de stratégies de transfusions restrictives, et quels en sont les risques? Il est bien entendu très difficile de répondre à ces questions, notamment en raison de l'hétérogénéité des études, des patients, des critères d'évaluation et des seuils de transfusion utilisés dans les études.

Bien qu'il ne s'agisse pas de la première méta-analyse abordant la question des seuils périopératoires de transfusion auprès des patients de chirurgie cardiaque, l'équipe de Kashani et coll. ${ }^{1}$ est l'une des premières à se servir de la technique d'analyse séquentielle d'études dans le cadre de leur méta-analyse. Bien qu'elle soit complexe d'un point de vue mathématique, la théorie sous-jacente à ce type d'analyse est simple : il s'agit d'une méta-analyse cumulative qui implique la création de limites séquentielles d'études pour définir la supériorité, l'infériorité ou la futilité. Si le résultat méta-analytique dépasse la limite de futilité, il est alors probable que d'autres études ne modifieraient pas le résultat global de la méta-analyse (de futures recherches seraient donc futiles). À l'inverse, si le résultat dépasse les limites de supériorité ou d'infériorité, une taille d'information (taille d'échantillon cumulée) et une signification statistique suffisantes ont été atteintes pour que les résultats soient considérés comme définitifs. Enfin, il est possible qu'aucun de ces seuils ne soit dépassé et que des recherches supplémentaires puissent potentiellement modifier le résultat méta-analytique. ${ }^{7}$

Fait intéressant, malgré les 9101 patients inclus dans l'analyse de Kashani et coll., ${ }^{1}$ seulement $30 \%$ de la taille d'information requise a été atteinte et aucun seuil de supériorité, d'infériorité ou de futilité n'a été dépassé. Du point de vue d'un clinicien, cela veut dire d'une part que d'autres études pourraient potentiellement démontrer des avantages supplémentaires en ce qui a trait à la mortalité et aux autres critères d'évaluation secondaires à l'étude. Par contre, tout avantage ou bienfait potentiel (s'il y en a) aura probablement une magnitude moindre, voire peut-être non significative d'un point de vue clinique. Ainsi, même en se fondant sur les données probantes, le message à retenir demeure inchangé : les patients en période périopératoire reçoivent moins de transfusions sanguines avec un seuil de transfusion restrictif $v s$ libéral - sans préjudice apparent aux devenirs cliniques.

Il est indubitable que les transfusions sanguines inutiles sont risquées - nous sommes tous très conscients des résultats cliniques négatifs potentiels et du coût des produits sanguins. Notre compréhension de cette question a été influencée par les scandales de sang contaminé des années 1980, à cause desquels des milliers de patients ont été accidentellement infectés par le VIH et/ou l'hépatite C. Ces incidents ont par la suite amélioré notre compréhension des devenirs immunologiques négatifs liés à la transfusion. Étant donné ce qui précède, comment faire alors pour avoir une pratique de transfusion fondée sur les données probantes? La question cruciale (et peut-être le Graal) de ce domaine de recherche serait d'identifier le véritable seuil à partir duquel une transfusion devient bénéfique pour chaque patient individuel. Voilà ce à quoi nous devrions aspirer: disposer d'un seuil à la fois personnalisé et contextuel à partir duquel nous saurions avec certitude qu'une transfusion apportera un avantage thérapeutique. Jusqu'à ce que nous parvenions à déterminer un tel seuil, nous continuerons probablement de transfuser inutilement certains patients et de ne pas transfuser d'autres patients qui en auraient bénéficié. Il va sans dire qu'un substitut artificiel et sécuritaire d'hémoglobine éliminerait une grande partie des angoisses associées à la transfusion d'érythrocytes; peut-être s'agit-il là du véritable Graal.

Cette étude importante nous démontre deux choses importantes, tant dans la vie que dans les sciences en général : parfois nous ne savons pas ce que nous ne savons pas, même si nous pensons savoir ce que nous savons. La conclusion classique d'une grande partie de notre littérature scientifique, selon laquelle «des recherches supplémentaires sont nécessaires », est omniprésente et frustrante pour tous ceux qui aspirent à la réponse définitive. Cette étude va peut-être un peu plus loin en suggérant que des recherches supplémentaires ne nous livreront probablement pas la réponse.

Conflicts of interest None.

Funding statement None.

Editorial responsibility This submission was handled by Dr. Philip M. Jones, Associate Editor, Canadian Journal of Anesthesia.

Conflit d'intérêt Aucun.

Déclaration de financement Aucune.

Responsabilité éditoriale Cet article a été traité par Dr Philip M. Jones, rédacteur adjoint, Journal canadien d'anesthésie.

\section{References}

1. Kashani HH, Lodewyks C, Kavosh MS, et al. The effect of restrictive versus liberal transfusion strategies on longer-term outcomes after cardiac surgery: a systematic review and meta- 
analysis with trial sequential analysis. Can J Anesth 2020; 67; DOI: https://doi.org/10.1007/s12630-020-01592-w.

2. Mazer CD, Whitlock RP, Ferguson DA, et al. Restrictive or liberal red-cell transfusion for cardiac surgery. N Engl J Med 2017; DOI: https://doi.org/10.1056/NEJMoa1711818.

3. Murphy GJ, Pike K, Rogers CA, et al. Liberal or restrictive transfusion after cardiac surgery. N Engl J Med 2015; 372: 9971008.

4. Shehata N, Mistry N, da Costa BR, et al. Restrictive compared with liberal red cell transfusion strategies in cardiac surgery: a meta-analysis. Eur Heart J 201; DOI: https://doi.org/10.1093/ eurheartj/ehy435.

5. Chen $Q H$, Wang $H L$, Liu L, et al. Effects of restrictive red blood cell transfusion on the prognoses of adult patients undergoing cardiac surgery: a meta-analysis of randomized controlled trials. Crit Care 2018; DOI: https://doi.org/10.1186/s13054-018-2062-5.

6. Kheiri B, Abdalla A, Osman M, et al. Restrictive versus liberal red blood cell transfusion for cardiac surgery: a systematic review and meta-analysis of randomized controlled trials. J Thromb Thrombolysis 2019; DOI: https://doi.org/10.1007/s11239-018-1784-1.

7. Thorlund K, Engstrøm J, Wetterslev J, et al. User Manual for Trial Sequential Analysis (TSA). Copenhagen: Copenhagen Trial Unit; 2017. Available from Copenhagen Trial Unit URL: http://www. ctu.dk/tsa/files/tsa_manual.pdf (accessed January 2020).

Publisher's Note Springer Nature remains neutral with regard to jurisdictional claims in published maps and institutional affiliations. 